\title{
Reversal agents of non-vitamin K dependent anticoagulants: a rapid review of the changing horizon
}

Supreet Kaur ${ }^{1}$, Abhishek Kumar ${ }^{1}$, Robert G. Lerner ${ }^{2}$, Wilbert S. Aronow ${ }^{3}$

\author{
${ }^{1}$ Department of Medicine, Saint Joseph Regional Medical Center, Paterson, \\ New Jersey, USA \\ 2Division of Hematology, Westchester Medical Center/New York Medical College, \\ Valhalla, NY, USA \\ ${ }^{3}$ Division of Cardiology, Westchester Medical Center/New York Medical College, \\ Valhalla, NY, USA
}

Submitted: 16 February 2016

Accepted: 27 March 2016

Arch Med Sci 2016; 12, 5: 1174-1178

DOI: 10.5114 /aoms.2016.61920

Copyright (c) 2016 Termedia \& Banach

\section{Abstract}

The newer non-vitamin K dependent anticoagulants (NOACs) have provided a new tool in the armamentarium of physicians treating nonvalvular atrial fibrillation and thromboembolism. Slowly, but steadily, there has been an increased preference of NOACs over vitamin K antagonists. However, the major limiting factor and the concern that precluded their use was lack of reversal in emergent situations. With the advent of reversal agents such as idarucizumab, andexanet alfa and PER977, this gap is also being filled. This will further increase the spectrum of usage of NOACs. In this review we present the detailed information on the completed trials on the reversal agents, the ongoinng trials, and their site of action. The reversal agent idarucizumab is FDA approved and readily available. The others are in clinical trials and are soon expected to be available in clinical practice.

Key words: non-vitamin K dependent anticoagulants, idarucizumab, andexanet alfa, PER977.

\section{Introduction}

Atrial fibrillation is the most common arrhythmia encountered in clinical practice [1]. The newer oral anticoagulants (NOAC) have emerged as safe, convenient and efficacious anticoagulants as compared to vitamin $\mathrm{K}$ antagonists [2]. Dabigatran, a direct thrombin inhibitor, was first approved by the FDA in 2010 for stroke prevention in non-valvular atrial fibrillation. Since then a number of other drugs in the class of factor Xa inhibitors including apixaban, rivaroxaban and edoxaban have been approved for not only stroke prevention but also for treatment and prevention of venous thromboembolic disease. Newer oral anticoagulants have been shown to be superior to warfarin in terms of predictable pharmacodynamics, fewer interactions with drugs and food and not requiring frequent monitoring [3].

Newer oral anticoagulants do have limitations, including limited ability to determine the blood level of the agents due to restricted availability of liquid chromatography tandem mass spectrometry (LC-MS/MS), which is the gold standard for measurement of plasma concentration of NOACs, especially in emergency situations $[4,5]$ and, until recently, a lack

\author{
Corresponding author: \\ Wilbert S. Aronow MD, FACC, \\ FAHA \\ Cardiology Division \\ New York Medical College \\ Macy Pavilion \\ Room 141 \\ Valhalla, NY 10595, USA \\ Phone: (914) 493-5311 \\ Fax: (914) 235-6274 \\ E-mail: wsaronow@aol.com
}


of specific reversal agents. Currently, non-specific supportive therapy including fresh frozen plasma and hemostatic factors such as prothrombin complex concentrates are being used in patients who are receiving NOACs and need emergent reversal of the anticoagulant effect. In this brief review, we will discuss specific reversal agents for NOAC anticoagulation.

Table I presents the completed trials on the reversal agents, and Table II shows the ongoing trials. Their site of action is shown in Figure 1.

\section{Idarucizumab (Praxbind)}

Idarucizumab, also known as BI 655075, was developed by Boehringer Ingelheim scientists in 2009. It is a humanized monoclonal antibody fragment derived by injecting mice with dabigatran-derived haptens coupled to carrier proteins. Its affinity to bind with dabigatran is approximately 350 times greater than thrombin [6]. It structurally mimics thrombin, but lacks a thrombin-like catalytic site and hence does not affect clotting in a diluted thrombin time assay. In animal models, idarucizumab showed rapid dose-dependent reversal of dabigatran for both internal and external bleeding $[6,7]$. Randomized double-blinded placebo controlled studies with healthy volunteers and with impaired renal function showed that intravenous idarucizumab reversed the anticoagulation effect of dabigatran within $5 \mathrm{~min}$, and multiple doses ( $2.5 \mathrm{~g}, 5 \mathrm{~g}$, and 2 times $2.5 \mathrm{~g}$ ) achieved sustained reversal $[8,9]$. Anticoagulation could be reestablished $24 \mathrm{~h}$ after the last dose of idarucizumab $[8,9]$.

Based on these promising results, a prospective multicenter cohort study was initiated to determine the Reversal effects of Idarucizumab on Active Dabigatran (RE-VERSE-AD, NCT02104947). A total of $5 \mathrm{~g}$ of idarucizumab (2.5 g of two doses not more than 15 min apart) was given to two groups [10]. Group A comprised patients with life-threatening or overt, uncontrollable bleeding (including intracranial hemorrhage, gastrointestinal hemorrhage, bleeding secondary to trauma, etc.), and group B comprised patients requiring emergent procedures (including acute fractures, acute appendicitis, acute cholecystitis, etc.). Interim analysis included 90 patients (51 in group A and 39 in group B). Idarucizumab completely reversed the anticoagulation effect of dabigatran in $88 \%$ to $98 \%$ of the patients who had elevated clotting times at baseline, with the median time to cessation of bleeding in group A being $11.4 \mathrm{~h}$. Only one patient reported an early $(<72 \mathrm{~h}$ after administration) thrombotic event including deep vein thrombosis and pulmonary embolism. Late events ( $>72 \mathrm{~h}$ after administration) included thrombotic events of deep vein thrombosis, pulmonary em-

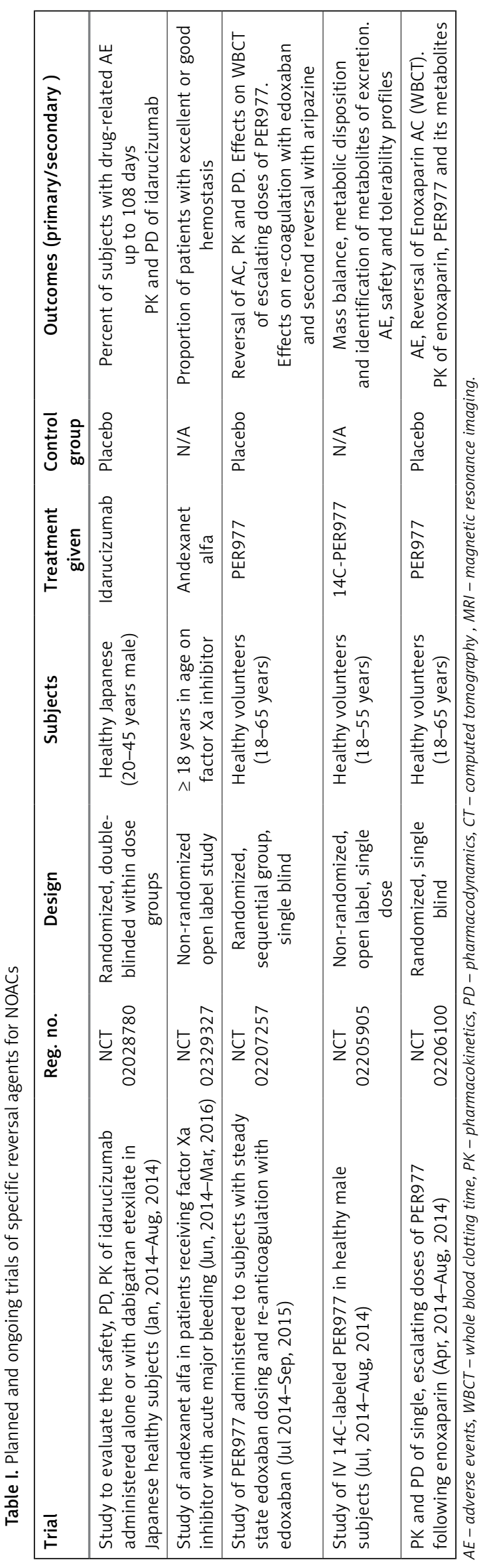




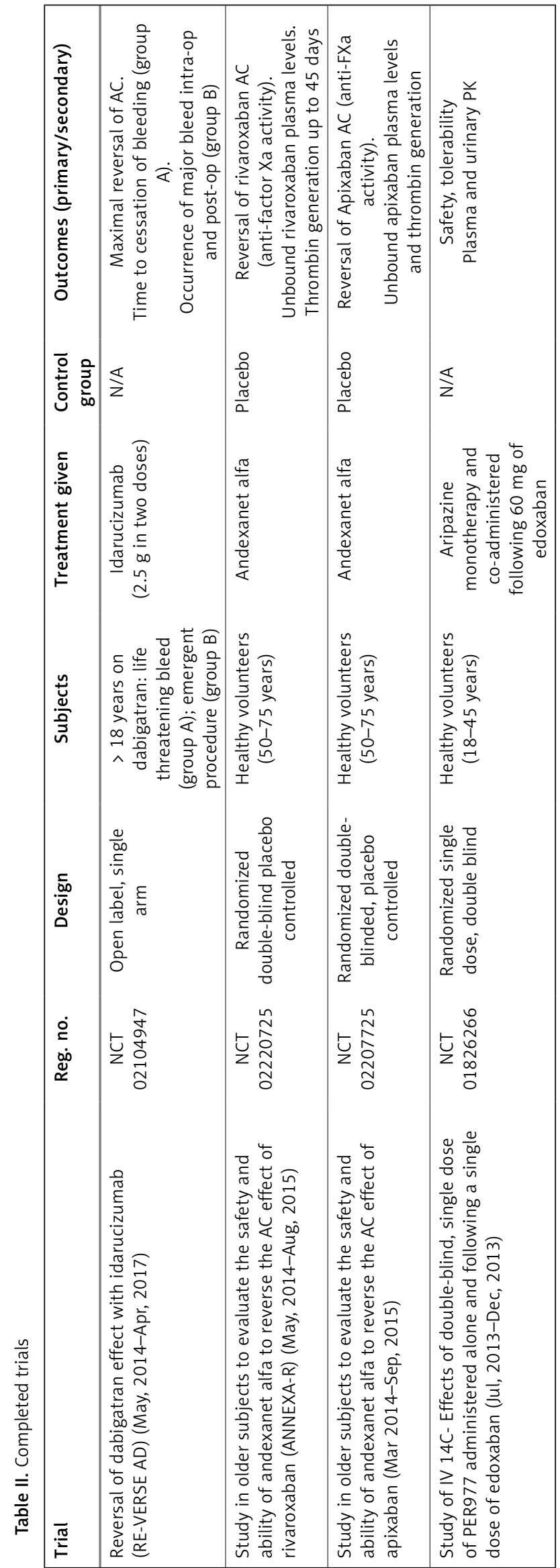

bolism, left atrial thrombus, ischemic stroke and non-ST elevation myocardial infarction. Other serious adverse effects included gastrointestinal hemorrhage, post-operative wound infection, delirium and pulmonary edema [10]. Idarucizumab is metabolized by biodegradation of the antibody into smaller peptides which are then reabsorbed. Its excretion is mainly via the kidneys, and no dose adjustment is needed in renally impaired patients. Following these results, idarucizumab was approved by the FDA in October, 2015 for reversal of dabigatran anticoagulant effects.

\section{Andexanet alfa}

Andexanet alfa (also known as PRT064445) is a modified factor Xa molecule which was developed by Portola pharmaceuticals for reversal of the anticoagulant effect of both direct and indirect factor Xa inhibitors. It is a modified recombinant protein (decoy protein) derived from human coagulation factor Xa that lacks a membrane binding domain and catalytic activity. Changing one serine to an alanine amino acid moiety makes it catalytically inactive while still maintaining high affinity for factor Xa inhibitors [11]. In a rivaroxaban treated rabbit liver laceration model, andexanet alfa reduced blood loss by $85 \%$ [11]. Crowther et al. observed that andexanet reversed the activity of rivaroxaban ex-vivo in healthy individuals in a dose-dependent manner [12]. In phase II studies, the effect of andexanet alfa was studied in healthy individuals with a follow-up of 48 days. These studies showed that andexanet reversed the effect of apixaban by $65 \%$, rivaroxaban by $20-53 \%$, and edoxaban by $52-73 \%$ without any major adverse effects $[13,14]$.

Randomized double-blind, placebo-controlled trials - Andexanet alfa, a Novel antidote to the anticoagulation Effects of factor Xa inhibitors Apixaban (ANNEXA-A) and Rivaroxaban (ANNEXA-R) - were initiated to determine the efficacy and safety of these reversal agents [14]. A total of 145 healthy older individuals, mean age 57.9 years, were assigned to either the apixaban or rivaroxaban group and received andexanet as a bolus of $400 \mathrm{mg}$ (part 1) or a bolus of $400 \mathrm{mg}$ followed by an infusion of $4 \mathrm{mg} /$ minutes for $120 \mathrm{~min}$ (part 2). In the apixaban group, anti-factor Xa activity was reduced by $94 \%$, and thrombin generation was fully restored in $100 \%$ of persons within 2 to $5 \mathrm{~min}$. In the rivaroxaban group, anti-factor Xa activity was reduced by $92 \%$, and thrombin generation was fully restored in $96 \%$ of persons [15]. The dose required for reversal of rivaroxaban was higher as compared to apixaban because of the higher initial maximum plasma concentration and larger volume of distribution of the former. There were no adverse effects such as thrombosis or 


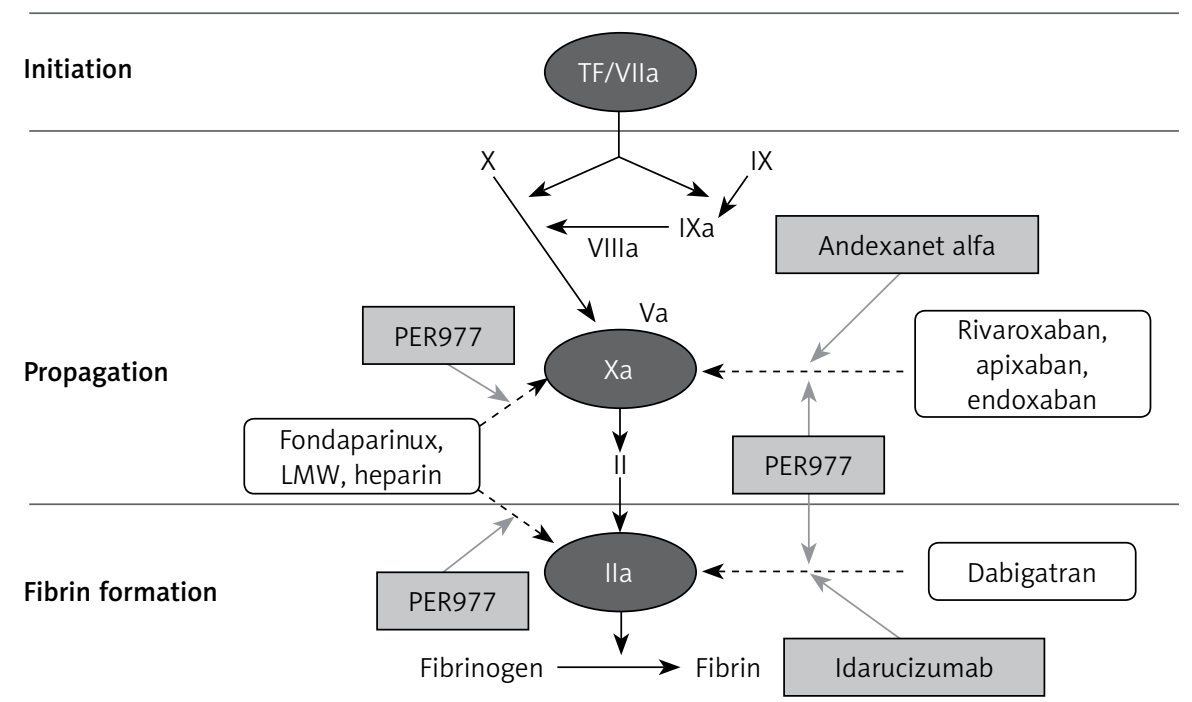

Figure 1. Mechanism of action and reversal agents for NOACS

bleeding noted. Non-neutralizing antibodies were detected in $17 \%$ of patients who received andexanet, indicating that it does have some immunogenicity. This study had the advantages that its participants were similar in age to those receiving factor Xa inhibitors in community practice and that widely accepted biomarkers of anticoagulation were used. Yet, further studies are needed on patients who require emergent or urgent reversal of factor Xa inhibitors.

\section{PER 977 (Aripazine)}

Aripazine (also known as PER977 or ciraparantag) is a small, synthetic, water-soluble, cationic molecule which was developed by Perosphere, Inc. to bind to unfractionated heparin and low molecular weight heparin through non-covalent hydrogen bonding and charge-charge interactions. In a similar manner, it has been shown to reverse the anticoagulant effects of factor $\mathrm{Xa}$ inhibitors and direct thrombin inhibitors $[16,17]$.

Aripazine significantly reduced bleeding (measured by blood loss volume) in a rat tail transection model treated with rivaroxaban, apixaban, edoxaban and dabigatran, while restoring all the laboratory indicators of an anticoagulant effect (prothrombin time, thromboelastography, activated partial thromboplastin time) to baseline within 20 min of administration [18]. To date, there is just one study of PER977 on healthy individuals dosed with edoxaban. In this study, subjects received a single intravenous dose of PER977 (100 mg to $300 \mathrm{mg}$ ) $3 \mathrm{~h}$ after administration of edoxaban. The baseline hemostasis as determined by whole blood clotting times was restored within 10 to $30 \mathrm{~min}$ and was sustained for $24 \mathrm{~h}$ [18]. Adverse effects included transient mild perioral and facial flushing and dysgeusia. No procoagulant activity was observed in the study [19].
In conclusion, information on pharmacokinetics and pharmacodynamics of andexanet alfa and aripazine is very limited because these drugs are in clinical trials involving healthy persons only. The half-life of idarucizumab is approximately $45 \mathrm{~min}$, that of andexanet alfa is $1 \mathrm{~h}$, and that of aripazine is $1.5 \mathrm{~h}$. The dose adjustment for idarucizumab in patients with liver disease has not been studied, and there is no dose adjustment needed for idarucizumab in patients with renal insufficiency. There are no contraindications for use of idarucizumab, but caution is advised in persons with hereditary fructose intolerance. Studies are underway to find similar data about pharmacokinetics and pharmacodynamics for newer reversal agents.

\section{Conflict of interest}

The authors declare no conflict of interest.

\section{References}

1. Kozlowski D, Budrejko S, Lip GY, et al. Lone atrial fibrillation: what do we now? Heart 2010; 96: 498-503.

2. Kozlowski D, Budrejko S, Raczak G, et al. Anticoagulant prevention in patients with atrial fibrillation: alternatives to vitamin K antagonists. Curr Pharm Des 2013; 19: 3816-26.

3. DeWald TA, Becker RC. The pharmacology of novel oral anticoagulants. J Thromb Thrombolysis 2014; 37: 217-33.

4. Blaich C, Müller C, Michels G, Wiesen MH. Multi-analytic analysis of non-vitamin $\mathrm{K}$ antagonist oral anticoagulants in human plasma using tandem mass spectrometry. Clin Chem Lab Med 2015; 53: 1981-90.

5. Douxfils J, Mani H, Minet V, et al. Non-VKA oral anticoagulants: accurate measurement of plasma drug concentrations. Biomed Res Int 2015; 2015: 345138.

6. Schiele F, van Ryn J, Canada K, et al. A specific antidote for dabigatran: functional and structural characterization. Blood 2013; 121: 3554-62.

7. Na SY, Mracsko E, van Ryn J, Veltkamp R. Idarucizumab improves outcome in murine brain hemorrhage related to dabigatran. Ann Neurol 2015; 78: 137-41. 
8. Glund S, Moschetti V, Norris S, et al. A randomised study in healthy volunteers to investigate the safety, tolerability and pharmacokinetics of idarucizumab, a specific antidote to dabigatran. J Thromb Haemost 2015; 113: 943-51.

9. Glund S, Stangier J, Schmohl M, et al. Safety, tolerability, and efficacy of idarucizumab for the reversal of the anticoagulant effect of dabigatran in healthy male volunteers: a randomised, placebo-controlled, double-blind phase 1 trial. Lancet 2015; 386: 680-90.

10. Pollack CV Jr, Reilly PA, Eikelboom J, et al. Idarucizumab for dabigatran reversal. N Engl J Med 2015; 373: 511-20.

11. Lu G, DeGuzman FR, Hollenbach SJ, et al. A specific antidote for reversal of anticoagulation by direct and indirect inhibitors of coagulation factor Xa. Nat Med 2013; 19: 446-51.

12. Crowther M, Kitt M, Lorenz T, et al. A phase 2 randomized, double-blind, placebo-controlled trial of PRT4445, a novel, universal antidote for direct and indirect factor Xa inhibitors. Presented at the XXIV Congress of the International Society on Thrombosis and Haemostasis Congress, Amsterdam, The Netherlands, July 2, 2013.

13. Mark C, Vandana M, Michael K, et al. A phase 2 randomized double- blind, placebo-controlled trial demonstrating reversal of rivaroxaban induced anticoagulation in healthy subjects by andexanet alfa (PRT06445): an antidote for FXa inhibitors. Blood 2013; 122: A3636.

14. Crowther M, Levy GG, Lu G, et al. A phase 2 randomized double-blind, placebo-controlled trial demonstrating reversal of edoxaban induced anticoagulation in healthy subjects by andexanet alfa (PRT06445): a universal antidote for factor Xa inhibitors. Blood 2014; 124: A4269.

15. Siegal DM, Curnutte JT, Connolly SJ, et al. Andexanet alfa for the reversal of factor Xa inhibitor activity. N Engl J Med 2015; 373: 2413-24.

16. Laulicht B, Bakhru S, Jiang X, et al. Antidote for new oral anticoagulants: mechanism of action and binding specificity of PER977. Presented at the $24^{\text {th }}$ Congress of the International Society on Thrombosis and Haematosis, Amsterdam, June 29-July 4, 2013.

17. Laulicht B, Bakhru S, Lee C, et al. Small molecule antidote for anticoagulants. Circulation 2012; 126: A11395.

18. Bakhru S, Laulicht B, Jiang X, et al. A synthetic small molecule which reverses over-dosage and bleeding by the new oral anticoagulants Ciuculation 2013; 128 : A18809.

19. Ansell JE, Bakhru SH, Laulicht BE, et al. Use of PER977 to reverse the anticoagulant effect of edoxaban. N Engl J Med 2014; 371: 2141-2. 